\title{
Editorial
}

\author{
Eugene N Myers \\ Distinguished Professor and Emeritus Chair \\ UPP Department of Otolaryngology \\ University of Pittsburgh Medical Center \\ Eye and Ear Institute, Suite-519, 200 Lothrop Street \\ Pittsburgh, PA 15213, USA \\ Phone: 412-647-2111, Fax: 412-647-2080 \\ e-mail: myersen@upmc.edu<mailto:myersen@upmc.edu
}

\section{Redefining the Role of the Head and Neck Surgeon in the Era of Chemoradiation}

Although there are now more than 500,000 cases of cancer of the head and neck worldwide each year, this was an uncommon disease until the second half of the 19th century when factories were built in Europe which could manufacture cigarettes cheaply. This led to widespread cigarette smoking and the increased prevalence of squamous cell carcinoma of the larynx, pharynx, and oral cavity. Increased availability of alcohol added yet another source of dangerous addiction.

Head and neck cancer has a significant impact on the quality of life both in form and function related to the tumor and the treatment. Historically, since surgical techniques, including total laryngectomy, resection of oral cavity cancer, and neck dissection were developed earlier than radiation therapy, surgical management of cancer of the head and neck was used exclusively for many years. ${ }^{1}$ However, surgery was dangerous and in the first half of the 20th century, radiation was often used as the first line treatment.

Following World War II, the availability of improved anesthesia techniques, blood banking, and antibiotics made it possible to use the existing surgical techniques safely. Many surgeons were attracted to the new specialty and came from all over the world to study with Dr. Hayes Martin, Chief of Head and Neck Surgery at Memorial Hospital and his successors, including Dr. Elliott Strong and Dr. Jatin Shah. Their strategy of an aggressive surgical approach became the predominant treatment paradigm nationally and internationally. Generations of head and neck surgeons were taught that the only way to manage the neck was by radical surgery and that radiation as a primary modality was unthinkable.

Conservation surgery of the larynx was practiced widely in the United States. Ogura popularized partial laryngeal surgery in the $1960 \mathrm{~s}^{2}$ Strong and Jako introduced transoral laser microsurgery for early glottic lesions in the 1970 s. $^{3}$ In Germany, Steiner and Rudert ${ }^{4}$ extended the use of laser microsurgery to include the excision of supraglottic and hypopharyngeal cancers. In France, Piquet then Laccourreye ${ }^{5}$ championed the use of supracricoid laryngectomy, which provided added versatility to the surgeon's approach to organ sparing laryngeal surgery.

As technology developed, cobalt took the place of radium and orthovoltage machines and provided a safer and more precise way of treating head and neck cancer with fewer complications and sequela. Its use as a single modality for cure for early cancer of the larynx, hypopharynx, and oropharynx and in an adjunctive mode following ablative surgery in advanced cancers became widely accepted.

The paradigm shift that led to redefining the role of the head and neck surgeon could be said to have started in 1845 with Peyrone who first described cisp $\mathrm{PtCl}_{2}\left(\mathrm{NH}_{3}\right)_{2}$ subsequently known as Peyrone's salt. In 1965, Barnett Rosenberg discovered that electrolysis of a platinum electrode produced cisplatin, which inhibited binary fission in Escherichia coli ( $E$. coli) bacteria. A series of experiments tested the effects of various platinum compounds on human leukemia and sarcoma cells and cis- $\mathrm{PtCl}_{2}\left(\mathrm{NH}_{3}\right)_{2}$ was found the most effective in killing these cells thereby setting the stage for the use of cisplatin in cancer treatment.

In 1973, Dr. Paul Chretian, a Surgical Oncologist at the National Institutes of Health (NIH), was using induction methotrexate for head and neck cancer. Some dramatic results in tumor reduction were noted but there were also some major complications. Dr. Lawrence Einhorn, at Indiana University, reported his successful use of cisplatinum in the treatment of 
nonseminoma testicular carcinoma. ${ }^{6}$ In 1978, Dr. Chretian and Dr. Robert Wittes, a Medical Oncologist, felt that the use of cisplatinum instead of methotrexate might increase the response rate and decrease the side effects.

This lead to the head and neck contracts program (HNCP 178) which began in 1978 with the participation of multiple institutions and cooperative groups. Dr. Gregory Wolf also played a leadership role in the program.

\section{CONCLUSIONS}

HNCP 178

- Induction cisplatinum was feasible

- High rates of complete response

- Responders did better than nonresponders

- Maintenance (adjuvant) chemotherapy was not feasible in this patient group

- No survival benefit.

The Department of Veterans Affairs study in 1991 led by Dr. Gregory Wolf changed the world for head and neck surgeons. ${ }^{7}$ The results, indicating that a high degree of organ preservation was achieved in the patients who had been treated with induction cisplatin, and radiation, were published in the New England Journal of Medicine, the highest profile US medical journal and were quickly picked up by prestigious newspapers such as the New York Times and the Wall Street Journal. This paradigm shift away from laryngectomy to chemoradiation was swift and dramatic. After all, when confronted with the choice of a laryngectomy with resultant loss of voice or chemoradiation with voice preservation and the same cure rate, who would not select chemoradiation?

In a follow-up study, RTOG 91-11 laryngeal preservation trial patients were randomized into induction chemotherapy + radiation or concurrent chemoradiotherapy or radiation alone. The best results were achieved with concurrent chemoradiation then induction chemotherapy followed by radiation and lastly radiation therapy alone. ${ }^{8}$

Criticisms of the VA study included: no radiation arm so that in RTOG-91-11 radiation alone was added; to surgery $X R T$, and chemoradiation. Neither the VA nor the RTOG protocol included patients with tumor invasion of the thyroid cartilage or extensive supraglottic tumors invading deep tongue musculature. Neither the VA nor the RTOG protocol included a conservation surgery arm.

In a fascinating cluster of the commentaries by Olsen, ${ }^{9}$ Wolf ${ }^{10}$ and Forastiere ${ }^{11}$ published in 2010 in Head and Neck, Wolf stated that the first goal of the chemoradiation strategy was to avoid total laryngectomy in a subset of patients who had cancer sensitive to chemotherapy. He quotes Chen as saying that there were no differences in survival between total laryngectomy and chemoradiation for Stage III laryngeal cancer patients. Not surprisingly, better survival was achieved with total laryngectomy in Stage IV patients. ${ }^{12}$

The VA study used tumor response to neoadjuvant chemotherapy as a biomarker to select patients for the study. That study and many that followed are remarkably consistent in identifying 65 to $70 \%$ of patients with radiation-sensitive tumors who can avoid total laryngectomy. With this biomarker, Dr. Wolf's Department at University of Michigan has achieved a remarkable long-term cause-specific survival rate of $87 \%$ in a nonselected series of patients with Stage III/ IV laryngeal cancer. ${ }^{10}$

The use of chemoradiation in oropharyngeal cancer has paralleled that of laryngeal cancer. The results in treating cancer of tonsil and base of the tongue have been dramatic. These, usually poorly differentiated squamous cell carcinomas, are exquisitely sensitive to the chemotherapy giving rapid cytoreduction which gives the radiation therapy a vastly decreased tumor volume to treat. The same protocol used in hypopharyngeal cancer has been less successful. In recent years, HPV has been implicated as a causative agent in the nonsmoker population of patients with cancer of the oropharynx. These patients, as a group, have a survival benefit when compared to the cohort of tobacco and alcohol users.

\section{Treatment strategies can now be tailored to the patient's needs and include:}

- Radiation therapy alone or radiation therapy combined with:

- Concurrent or induction chemotherapy with salvage surgery

- Targeted therapy, such as Erbitux

- Conservation surgical procedures

- Total laryngectomy.

How do We define the Current Role of the Head and Neck Surgeon in the Era of Chemoradiation

The surgeon must continue to be the captain of the head and neck team. He must continue to serve as the gate keeper, operate when it is the best treatment for patients and remain engaged in the patients care even when they choose chemoradiation. The traditional role of the head and neck surgeon, including evaluation of the patient, pre-, intra-, and postoperative management, oversite of adjunctive treatment, and rehabilitative programs must be maintained. Naturally, the head and neck surgeon will continue to pursue the 
traditional surgery for tumors of the oral cavity, thyroid, salivary gland, paragangliomas, and skull base surgery.

Head and neck cancer surgery should be carried out in a university, university-affiliated or major tertiary hospital with a high volume of head and neck patients. Chen et al studied the impact of the treating facilities volume of cases on survival for early stage laryngeal cancer to evaluate the relationship between total hospital treatment volume and survival in the first five years after treatment. Their analysis revealed that for patients with early stage cancer of the larynx, several factors are associated with improved survival, including type of treatment, volume of cases in the treating facilities, and the patient's insurance status. The report also indicated that patients with early stage laryngeal cancer had a substantially better survival when treated with surgery than those treated initially with chemoradiation. ${ }^{12}$

The head and neck surgeon must continue to be involved in the diagnosis and staging of the tumor and appropriate evaluation of comorbidities. Advanced imaging studies, such as PET-CT scanning have been very useful in staging and followup in patients treated with chemoradiation. Patients with airway obstruction will need a tracheostomy. This, of course, is a role the surgeons must play since the medical and radiation oncologists are not qualified to evaluate and manage the airway.

The surgeon must evaluate the patient's nutritional status. Nutritional status is a very important predictive factor for response to chemoradiation. Many patients in developing countries are malnourished as baseline and made worse by dysphagia superimposed by the tumor and its treatment. Every effort, including PEG tubes, should be used in nutritional replenishment since poor nutritional status usually leads to poor response to chemoradiation as well as delayed healing in those patients who come to surgery either as primary or salvage surgery.

The surgeon is the individual really responsible for continuity of care for head and neck patients using consultants in other specialties as deemed necessary. The surgeon should monitor the progress of the patient during chemoradiation therapy. If the tumor is not responding, surgery should be advised. During the course of treatment, the surgeon's continuous attention to provide an adequate airway, adequate state of hydration, and nutritional needs is essential. Some patients will require hospitalization to stabilize their condition.

Initially, the radical neck dissection was the only treatment for metastatic cancer in the neck. The management of the neck has undergone an evolution in the chemoradiation era. In the early days of chemoradiation, patients with N2-3 metastatic cancer underwent planned radical neck dissection approximately 6 weeks postchemoradiation. The use of PET CT to monitor these patients is now usually carried out 3 months postchemoradiation which gives a more precise indication as to whether complete locoregional control is established or not. In cases with limited residual nodal disease, a selective neck dissection or a superselective neck dissection has been a successful mean in rendering these patients to be disease free. ${ }^{13,14}$ Patients with a complete response in the neck will not require neck dissection.

The head and neck surgeon still carries the burden of responsibility for the management of early, late, and recurrent squamous cell carcinoma. In 2006, a panel of experts was convened by the American Society of Clinical Oncology (ASCO) to develop clinical guidelines for larynx preservation strategies. Their recommendations were for organ preservation surgery for T1-T2 lesions mostly using transoral laser surgery, and voice preservation for using chemoradiation for T3 or T4 without tumor invasion into soft tissues with total laryngectomy reserved for more advanced T4 lesions. Chen found that total laryngectomy was associated with increased survival compared with radiation or chemoradiation for Stage IV cancer. ${ }^{15}$

Robotic surgery (TORS) has been used successfully in excising tumors from the larynx, oropharynx, nasopharynx and skull base. Despite the initial expense of the instrument, it is our expectation that the approach will be adapted for cases which today would be subjected to chemoradiation. Genden et $\mathrm{al}^{16}$ uses TORS for the excision of cancer of the base of the tongue or tonsil with neck dissection followed by a lower dose of XRT. This dose de-escalation results in cure rates similar to chemoradiation but with less fibrosis and dysphagia.

Salvage surgery for recurrent or persistent cancer in those patients treated with chemoradiation therapy brings a formidable challenge to the head and neck surgeon. The VA study revealed that approximately two-thirds of the patients, treated with chemoradiation, were disease free after two years of follow-up. Thus, one-third of the patients so treated will be potential subjects for salvage surgery. Similarly, the patients who fail chemoradiation for hypopharyngeal cancer, which is a higher percentage than Stage III-IV laryngeal cancer and oropharyngeal cancer, will be candidates for salvage surgery.

The surgeon must re-evaluate the patient postchemoradiation. If there is evidence of residual primary cancer, biopsy and salvage surgery is indicated. Salvage surgery in the postchemoradiation therapy patient may be challenging. 
Delayed healing related to patient's nutritional status and the effect of the chemoradiation therapy on the tissues results in a high rate of fistula formation following total laryngectomy. In the RTOG-91-11, ${ }^{8}$ the fistula rate was $30 \%$ for chemoradiation compared to $15 \%$ for XRT alone. The use of microvascular free tissue transfer in salvage total laryngectomy either in primary closure or as an additional layer to bolster the primary closure has proved very effective in decreasing the fistula rate resulting in early restoration of swallowing, earlier discharge and decreased hospital costs. ${ }^{17}$

Many patients will develop dysphagia from fibrosis of the pharyngeal constrictor muscles. The head and neck surgeon will be able to help many of these patients by carrying out serial dilatations of the pharynx, but others, especially those treated for laryngeal, hypopharyngeal and oropharyngeal cancer, will have a permanent gastrostomy tube. While many of the patients treated with chemoradiation in an attempt at organ preservation will be cured, not all will be functional. Some patients will develop fibrosis of the larynx requiring a tracheostomy and a feeding tube or chondronecrosis of the laryngeal cartilage. Both situations will require a total laryngectomy. My suggestion to the head and neck surgeon is "do not put away your knives."

Most patients with terminal cancer will seek the help of the surgeon and may need hospitalization for the care of fungating tumors, pain management, control of bleeding and some hand holding and consolation at the end.

\section{Advances in therapy which have contributed to a redefinition of the role of the surgeon:}

- The use of chemotherapeutic agents in combination with radiation therapy.

- Advances in radiation therapy, such as IMRT and Cyberknife.

- Advances in medical oncology and molecular targeted therapy including targeted therapy.

- Advances in diagnosis, staging, and surveillance using PETCT scanning.

- New technical advances in surgical therapy, such as robotics.

Individualized cancer therapy in the future will be driven by molecular staging and treatment. Emphasis on education and proper training of our head and neck surgeons to be the leaders of the team, the proliferation of surgeon-scientists and emphasis on taking our clinical problems to the laboratory and the laboratory findings to the clinic will be the solution for patients with head and neck cancer in future.
These oft-quoted organ preservation protocols have been developed in the United States and Western Europe where modern equipment and well-trained professionals are abundant. Problems arise with the application of organ preservation strategies in developing countries due to critical factors, such as advanced stage cancers, comorbidities, poor nutrition, long distance to travel and other barriers to access, such as poverty, lack of facilities or availability of chemotherapy and radiation therapy, difficulty in adhering to protocol standards and lack of expertise in performing salvage surgery. ${ }^{18}$

\section{REFERENCES}

1. Myers E. History and Evolution of Head and Neck Oncology. In: de Souza C (Ed). Head and Neck Surgery. New Dehli: India Jaypee Brothers Medical Publishers, 2009:601-16.

2. Ogura J, Biller H. Conservation surgery in cancer of the head and neck. Otolaryngol Clin North Am 1969;2:641-65.

3. Strong $M$, Jako $G$. Laser surgery in the larynx. Early clinical experience with continuous $\mathrm{CO}_{2}$ laser. Ann Otol Rhinol Laryngol 1972;81:791-98.

4. Rudert H, Werner J. Endoscopic resections of glottic and supraglottic carcinomas with the $\mathrm{CO}_{2}$ laser. Eur Arch Otorhinolaryngol 1995;252:146-48.

5. Laccourreye O, Muscatello L, Laccourreye L, et al. Supracricoid partial laryngectomy with cricohyoidoepiglottopexy for "early" glottic carcinoma classified as T1-T2NO invading the anterior commissure. Am J Otolaryngol 1997;18:385-90.

6. Einhorn L, Williams S. Chemotherapy of disseminated seminoma. Cancer Clin Trials 1980;3:307-13.

7. Wolf G, Hong W, Fisher S, et al. The Department of Veterans Affairs Laryngeal Cancer Study Group. Induction chemotherapy plus radiation compared to surgery plus radiation in patients with advanced laryngeal cancer. N Engl J Med 1991;324: 1685-90.

8. Forastiere AA, Goepfert H, Maor M, Pajak TF, Weber R, Morrison W, et al. Concurrent chemotherapy and radiotherapy for organ preservation in advanced laryngeal cancer. N Engl J Med 2003;349(22):2091-98. Available from http://www.ncbi. nlm.nih.gov/entrez/query.fcgi? cmd = Retrieve and $\mathrm{db}=$ PubMed \& dopt $=$ Citation \& list_uids $=14645636$.

9. Olsen KD. Re-examining the treatment of advanced laryngeal cancer. Head Neck 2010;32(1):1-7. Available from http:// www.ncbi.nlm.nih.gov/entrez/query.fcgi cmd $=$ Retrieve $\& d b$ $=$ PubMed\&dopt $=$ Citation\&list_uids $=19953627$.

10. Wolf G. Re-examining the treatment of advanced laryngeal cancer: The VA Laryngeal Cancer Study Revisited. Head and Neck 2010;32:7-14.

11. Forastiere A. Larynx preservation and survival trends: Should there be concern? Head and Neck 2010;32:14-17.

12. Chen A, Pavluck A, Halpern M, Ward E. Impact of treating facilities' volume on survival for early-stage laryngeal cancer. Head and Neck 2009;31(9):1137-43. 
13. Lango M, Andrews G, Ahmad S, Feigenberg S, Tuluc M, Gaughan $\mathrm{J}$, et al. Postradiotherapy neck dissection for head and neck squamous cell carcinoma: Pattern of pathologic residual carcinoma and prognosis. Head and Neck 2009;31:328-37.

14. Vedrine PO, Thariat J, Hitier M, Janot F, Kaminsky MC, Makeieff $M$, et al. Need for neck dissection after radiochemotherapy? A study of the French GETTEC Group. Laryngoscope 2008;118(10):1775-80. Available from http://www.ncbi.nlm. nih.gov/entrez/query.fcgi?cmd = Retrieve\&db = PubMed\&dopt $=$ Citation\&list_uids $=18806474$.

15. Chen A, Halpern H. Factors predictive of survival in advanced laryngeal cancer. Arch Otolaryngol Head and Neck Surg 2007;133:1270-76.
16. Genden E. Transoral Robotic Surgery: Outcomes and Future Directions 4th World Congress of International Federation of Head and Neck Oncologic Societies Seoul, Korea, 2010:24.

17. Teknos T, Myers L, Bradford C, Chepeha D. Free tissue reconstruction of the hypopharynx after organ preservation therapy: Analysis of wound complications. Laryngoscope 2001;111(7):1192-96.

18. Sanabria A, Domenge C, D’Cruz A, Kowalski LP. Organ preservation protocols in developing countries. Curr Opin Otolaryngol Head Neck Surg 2010;18(2):83-88. Available from http://www.ncbi.nlm.nih.gov/entrez/query.fcgi?cmd = Retrieve\&db = PubMed\&dopt $=$ Citation\&list_uids $=20216217$. 